\title{
The cyclic homology of affine algebras
}

\author{
Ioannis Emmanouil \\ Department of Mathematics, The University of Michigan, Ann Arbor, MI 48109, USA
}

Oblatum 25-IV-1994 \& 21-XI-1994

\begin{abstract}
In this paper, we study the cyclic homology of affine algebras over a field of characteristic 0 . We show that if $A$ is such an algebra the inverse system $\left(H C_{*+2 m}(A), S\right)_{m}$ decomposes in sufficiently large degrees into the direct sum of the constant system with value $\bigoplus_{i \in \mathbf{Z}} H_{\text {inf }}^{*+2 l}(A)$ and a system which is essentially zero. The essentially zero component is the kernel of the LodayQuillen map $\mu$ and the behavior of the restriction of $S$ on it is closely related to the degeneracy of the spectral sequence associated with Connes' exact couple of $A$.
\end{abstract}

\section{Introduction}

It is well-known that the cyclic homology of the algebra $A$ of regular functions on an affine algebraic variety over a field of characteristic 0 is closely related to the cohomology of the variety. In the smooth case, this relation is expressed by the existence of an isomorphism

$$
\mu_{n}: H C_{n}(A) \longrightarrow \Omega_{A}^{n} / d \Omega_{A}^{n-1} \oplus H_{\mathrm{dR}}^{n-2}(A) \oplus H_{\mathrm{dR}}^{n-4}(A) \oplus \cdots
$$

defined by Loday and Quillen in [19]. (The continuous analogue of this result was already obtained by Connes, who introduced in [5] the cyclic (co-) homology of an associative algebra over the complex numbers as an analogue of the de Rham cohomology of a space in the context of his noncommutative geometry.) The map $\mu$ is defined for non-smooth algebras as well, where $H_{\mathrm{dR}}(A)$ is the cohomology of the de Rham (Kähler) complex $\Omega_{A}^{*}$, but it is not always an isomorphism. In the general case, the relation between the cyclic homology of $A$ and the cohomology of $\operatorname{Spec} A$ can be better expressed in terms of the periodic cyclic homology, a 2-periodic version of cyclic homology obtained by inverting an endomorphism $S$ of the latter of degree -2 . According 
to a theorem due to Feigin and Tsygan (cf. [8]), the periodic cyclic homology $H P(A)$ of $A$ is isomorphic with the infinitesimal cohomology $H_{\text {inf }}(A)$ of $\operatorname{Spec} A$, viewed with the even-odd grading. This theorem can be proved following the approach of Deligne and Hartshorne to infinitesimal cohomology (cf. [13]), where one embeds a given variety as a closed subvariety of a smooth variety and then considers the completion of the de Rham complex of the latter along the subvariety. This method was developed by Wodzicki, Seibt and Hübl (cf. $[14,24,27])$ and led to a generalization of the above result to the case where the ground ring is any commutative Noetherian $\mathbf{Q}$-algebra.

The cyclic homology $H C(A)$ admits a functorial decomposition which can be described using the non-abelian derived functor approach of $[4,8]$ or the combinatorial approach of $[9,17,21]$. There is a corresponding decomposition of the periodic cyclic homology $H P(A)$ into the direct product of components $H P^{(i)}(A), i \in \mathbf{Z}$, and the isomorphism of Feigin and Tsygan decomposes into the direct product of isomorphisms $H P_{*}^{(i)}(A) \simeq H_{\mathrm{inf}}^{2 i-*}(A)$.

An auxiliary tool for understanding the action of $S$ on $H C(A)$ is the spectral sequence associated with Connes' exact couple of $A$. We show that the limit term $E^{\infty}$ of that spectral sequence is isomorphic with the infinitesimal cohomology $H_{\text {inf }}(A)$; this proves a conjecture of Wodzicki. The idea of the proof is to show that the spectral sequence converges to periodic cyclic homology. Then, $E^{\infty}$ is the graded vector space associated with a certain filtration of $H P(A)$. Using Deligne's identification of infinitesimal cohomology with singular cohomology over $\mathbf{C}$ and a result of Bloom and Herrera, we show that this associated graded vector space coincides with $H_{\text {inf }}(A)$. The convergence of the spectral sequence follows since the inverse system $\left(H C_{*+2 m}(A), S\right)_{m}$ satisfies the Mittag-Leffler condition, a fact which is itself proved using the finiteness of infinitesimal cohomology. The initial term of the spectral sequence contains the de Rham (Kähler) cohomology $H_{\mathrm{dR}}(A)$ and the canonical map $H_{\mathrm{inf}}^{\mathrm{n}}(A) \longrightarrow$ $H_{\mathrm{dR}}^{n}(A)$ is identified with an edge homomorphism. Hence, the intermediate terms of the spectral sequence should be viewed as higher order approximations of $H_{\text {inf }}(A)$.

Combining the above results, we obtain a fairly complete picture about the asymptotic structure of the inverse system $\left(H C_{*+2 m}(A), S\right)_{m}$. In order to state our main result, let us denote by $V_{n}(A)$ the kernel of the Loday-Quillen map $\mu_{n}$; then, $V(A)=\bigoplus_{n \geqq 0} V_{n}(A)$ is an $S$-invariant subspace of $H C(A)$. We prove the following

Theorem The inverse system $\left(H C_{*+2 m}(A), S\right)_{m}$ decomposes in sufficiently large degrees into the direct sum of the constant system with value $\bigoplus_{l \in \mathbf{Z}} H_{\mathrm{inf}}^{*+2 l}(A)$ and the system $\left(V_{*+2 m}(A), S\right)_{m}$, which is essentially zero.

The behavior of $S$ on $V(A)$ determines the degeneracy of the spectral sequence associated with Connes' exact couple of $A$; we show that the restriction $\left.S\right|_{V(A)}$ is nilpotent if and only if the spectral sequence degenerates. 
In order to justify the plausibility of this criterion, we note that the differential $\partial^{r}$ of the spectral sequence is closely related to the iterate $S^{r}$. Hence, the nilpotence of $S$ on $V(A)$ essentially means that the component $V(A)$ of $H C(A)$ does not affect the behavior of the spectral sequence in sufficiently large degrees.

These results were announced in a Note to Comptes Rendus [7].

The contents of this work are as follows:

Section 1 is introductory. We set up the notation we will be using and recall the basic facts about the Hochschild and cyclic homology of algebras. We also review the decomposition of the homology of commutative algebras and record the properties that will be needed. The reader can find all these notions and prerequisite results in Loday's book [18].

In Sect. 2, we first detail the decomposition of the periodic cyclic homology of commutative algebras and prove that the components of this decomposition can be identified, for an affine algebra, with infinitesimal cohomology. For such an algebra, we then prove that the inverse system $\left(H C_{*+2 m}, S\right)_{m}$ satisfies the Mittag-Leffler condition, using a general property of inverse systems of countably generated vector spaces. We also show that the infinitesimal cohomology admits canonical embeddings into the components of cyclic homology.

In Sect. 3, after some generalities about the spectral sequence associated with an exact couple, we consider the case of Connes' exact couple and prove that the limit term of the corresponding spectral sequence is isomorphic, for an affine algebra, with infinitesimal cohomology. As an application, we then prove our main result about the structure of the inverse system $\left(H C_{*+2 m}, S\right)_{m}$. Finally, we examine the relation between the degeneracy of the spectral sequence and the kernel of the Loday-Quillen map $\mu$.

For the sake of completeness, we have included an appendix with some results about inverse limits that are used in the main text.

Notations and terminology. Unless otherwise specified, $k$ will be a fixed field of characteristic 0 and all vector spaces and algebras will be over $k$. By a (double) complex we will mean a (double) chain complex. If $(C, \partial)$ is a complex and $m \in \mathbf{Z}$, we will denote by $(C[m], \partial[m])$ the complex with $(C[m])_{n}=C_{n-m}$ and $(\partial[m])_{n}=(-1)^{m} \partial_{n-m}$.

\section{Prerequisites}

\subsection{Cyclic homology of algebras}

Let $A$ be an algebra and consider the normalized Hochschild complex ( $C(A), b)$, whose homology is the Hochschild homology $H H(A)$ of $A$. Recall that $C_{n}(A)=$ $A \otimes \bar{A}^{\otimes n}$, where $\bar{A}=A / k$ and the tensor products are over $k$. The complex $(C(A), b)$ can be endowed with the structure of a mixed complex by means 
of certain maps $B: C_{n}(A) \longrightarrow C_{n+1}(A)$ satisfying the relations $B^{2}=0$ and $B b+b B=0$ (cf. $[15,19]$ ).

We now consider the double complex $\mathscr{B}(A)$ defined by letting the component of degree $(p, q)$ be $C_{q-p}(A)$ if $p, q \geqq 0$ and the horizontal (resp. vertical) differential be $B$ (resp. $b$ ). The homology of the associated total complex is the cyclic homology $H C(A)$. The short exact sequence of complexes

$$
0 \longrightarrow(C(A), b) \stackrel{I}{\longrightarrow} \text { Tot } \mathscr{B}(A) \stackrel{s}{\longrightarrow}(\operatorname{Tot} \mathscr{B}(A))[2] \longrightarrow 0,
$$

where $S$ is obtained by omitting the first column of $\mathscr{B}(A)$, induces a functorial long exact sequence (Connes' exact sequence)

$$
\cdots \stackrel{B}{\longrightarrow} H H_{n}(A) \stackrel{I}{\longrightarrow} H C_{n}(A) \stackrel{S}{\longrightarrow} H C_{n-2}(A) \stackrel{B}{\longrightarrow} H H_{n-1}(A) \stackrel{!}{\longrightarrow} \cdots
$$

In other words, there is an exact couple $\mathscr{E}(A)$ (Connes' exact couple; cf. [5])

$$
\begin{gathered}
H C(A) \stackrel{S}{\longrightarrow} H C(A) \\
\qquad \swarrow^{B}(A)
\end{gathered}
$$

The inverse limit of the inverse system $((\operatorname{Tot} \mathscr{B}(A))[-2 m], S)_{m}$ is the complex associated with the double complex $\mathscr{B}_{\text {per }}(A)$ whose component of degree $(p, q)$ is $C_{q-p}(A)$ for all $p, q \in \mathbf{Z}$ with horizontal (resp. vertical) differential given by $B$ (resp. $b$ ). The homology of that complex is the periodic cyclic homology $H P(A)$; it is related to cyclic homology by the short exact sequence

$$
0 \longrightarrow \lim _{m}^{\prime} H C_{*+2 m+1}(A) \longrightarrow H P_{*}(A) \longrightarrow \lim _{m} H C_{*+2 m}(A) \longrightarrow 0
$$

Now suppose that $A$ is commutative and consider the double complex $\mathscr{D}(A)$ consisting of $\Omega_{A}^{q-p}$ in degree $(p, q)$ if $p, q \geqq 0$ with horizontal (resp. vertical) differential given by $d=d_{\mathrm{dR}}$ (resp. 0). The maps $\mu_{n}: C_{n}(A) \longrightarrow \Omega_{A}^{n}$ with $a_{0} \otimes a_{1} \otimes \cdots \otimes a_{n} \mapsto 1 / n ! a_{0} d a_{1} \cdots d a_{n}$ induce a morphism of double complexes $\mu: \mathscr{B}(A) \longrightarrow \mathscr{D}(A)$; this is the Loday-Quillen map. If $A$ is smooth, $\mu$ is a quasi-isomorphism (cf. [19]).

\subsection{Decompositions for commutative algebras}

We now fix a commutative algebra $A$. In this case, $H H(A)$ and $H C(A)$ admit functorial decompositions, which can be described by the combinatorial approach of $[9,17,21]$ as follows. Viewing the components of the normalized Hochschild complex as modules over the corresponding symmetric groups and using a certain family of idempotents of the associated rational group rings, one obtains a decomposition $(C(A), b)=\bigoplus_{i \geqq 0}\left(C^{(i)}(A), b\right)$, with $C_{n}^{(i)}(A)=0$ 
for $i>n$. Hence, there results a decomposition of $H H(A)$; we have

$$
H H_{n}(A)=\bigoplus_{l \geq 0} H H_{n}^{(l)}(A)=\bigoplus_{\imath=0}^{n} H H_{n}^{(\imath)}(A),
$$

where $H H_{n}^{(I)}(A)=H_{n}\left(C^{(i)}(A)\right)$.

The operator $B$ maps $C^{(i)}(A)$ into $C^{(i+1)}(A)$ and hence the double complex $\mathscr{B}(A)$ decomposes as $\mathscr{B}(A)=\bigoplus_{i \geqq 0} \mathscr{B}^{(i)}(A)$, where $\mathscr{B}^{(i)}(A)$ is the subcomplex of $\mathscr{B}(A)$ consisting of $C_{q-p}^{(t-p)}(A)$ in degree $(p, q)$ if $p, q \geqq 0$. There results a decomposition of $H C(A)$; we have

$$
H C_{n}(A)=\bigoplus_{i \geqq 0} H C_{n}^{(i)}(A)=\bigoplus_{i=0}^{n} H C_{n}^{(i)}(A)
$$

where $H C_{n}^{(\ell)}(A)=H_{n}\left(\operatorname{Tot} \mathscr{B}^{(i)}(A)\right)$.

The short exact sequence (1) decomposes into the direct sum of

$$
0 \longrightarrow\left(C^{(i)}(A), b\right) \stackrel{I}{\longrightarrow} \operatorname{Tot}: \mathscr{B}^{(i)}(A) \stackrel{S}{\longrightarrow}\left(\operatorname{Tot} \mathscr{B}^{(i-1)}(A)\right)[2] \longrightarrow 0, \quad i \geqq 0 .
$$

Hence, Connes' exact sequence admits itself a decomposition into the direct sum of exact sequences of the form

$$
\cdots \stackrel{B}{\longrightarrow} H H_{n}^{(i)}(A) \stackrel{l}{\longrightarrow} H C_{n}^{(i)}(A) \stackrel{S}{\longrightarrow} H C_{n-2}^{(t-1)}(A) \stackrel{B}{\longrightarrow} H H_{n-1}^{(t)}(A) \stackrel{\perp}{\longrightarrow} \cdots
$$

Remark 1.1 The above decompositions can be also described using the non-abelian derived functor approach of $[4,8]$. The equivalence of the two approaches is shown in $[17,26]$ (see also [22]).

The map $\mu_{n}: C_{n}(A) \longrightarrow \Omega_{A}^{n}$ of Sect. 1.1 vanishes on $\bigoplus_{l=0}^{n-1} C_{n}^{(i)}(A)$ and induces an isomorphism $\mu_{n}: H H_{n}^{(n)}(A) \stackrel{\sim}{\longrightarrow} \Omega_{A}^{n}$. Let $\mathscr{O}^{(i)}(A)$ be the double complex consisting of the truncated de Rham complex

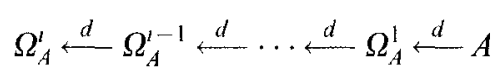

in the $i$ th row and zeroes elsewhere. The map $\mu: \mathscr{B}(A) \longrightarrow \mathscr{D}(A)$ decomposes into the direct sum of $\mu^{(i)}: \mathscr{B}^{(i)}(A) \longrightarrow \mathscr{P}^{(i)}(A), i \geqq 0$. The induced maps in homology are compatible with $S$; in particular, for $i<n$ there is a commutative diagram

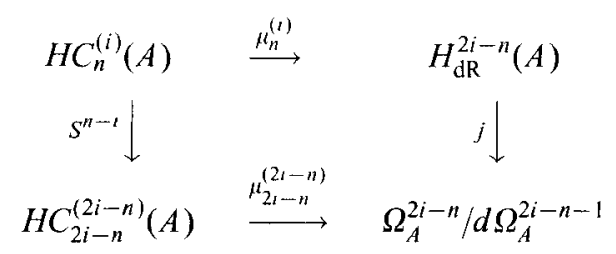


where $j$ is the inclusion. Moreover, $\mu_{n}^{(n)}: H C_{n}^{(n)}(A) \longrightarrow \Omega_{A}^{n} / d \Omega_{A}^{n-1}$ is an isomorphism for all $n$.

\section{Periodic cyclic homology and infinitesimal cohomology}

\subsection{The decomposition of periodic cyclic homology}

Let $A$ be a commutative algebra and consider the decompositions $C(A)=$ $\bigoplus_{i \supseteq 0} C^{(i)}(A)$ and $\mathscr{B}(A)=\bigoplus_{i \geqq 0} \mathscr{B}^{(i)}(A)$ described in Sect. 1.2. For $i \in \mathbf{Z}$, the inverse limit of the inverse system $\left(\left(\operatorname{Tot} \mathscr{B}^{(i+m)}(A)\right)[-2 m], S\right)_{m}$ is the complex associated with the double complex $\mathscr{B}_{\text {per }}^{(i)}(A)$ pictured below

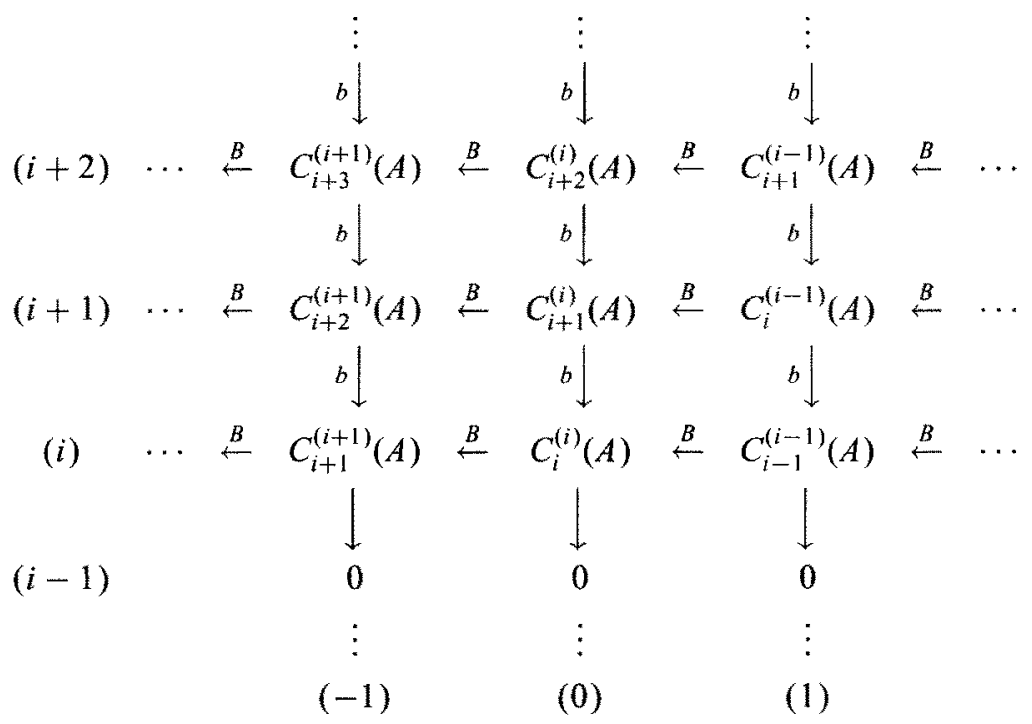

Let $H P^{(i)}(A)$ be the homology of $\operatorname{Tot} \mathscr{B}_{\mathrm{per}}^{(i)}(A)$. Since $\mathscr{B}_{\mathrm{per}}(A)=\Pi_{i \in \mathbf{Z}} \mathscr{B}_{\mathrm{per}}^{(i)}(A)$, we have $\operatorname{Tot} \mathscr{B}_{\text {per }}(A)=\prod_{i \in \mathbf{Z}} \operatorname{Tot} \mathscr{B}_{\text {per }}^{(i)}(A)$; hence, $H P(A)=\prod_{i \in \mathbf{Z}} H P^{(i)}(A)$ (cf. [17, Remark 4.10]).

We also consider the double complex $\mathscr{D}_{\mathrm{per}}^{(i)}(A)$ consisting of the de Rham complex

$$
\begin{aligned}
& \cdots \stackrel{d}{\longleftarrow} \Omega_{A}^{i+1} \stackrel{d}{\longleftarrow} \Omega_{A}^{i} \stackrel{d}{\longleftarrow} \Omega_{A}^{i-1} \stackrel{d}{\longleftarrow} \quad \ldots \\
& (-1) \quad(0) \quad \text { (1) }
\end{aligned}
$$

in the $i$ th row and zeroes elsewhere. For all $i \in \mathbf{Z}$, there is an induced morphism of double complexes $\mu^{(i)}: \mathscr{B}_{\text {per }}^{(i)}(A) \longrightarrow \mathscr{D}_{\text {per }}^{(i)}(A)$.

Now suppose that $A=R / I$, for some commutative algebra $R$ and some ideal $I \subseteq R$. Consider the $I$-adic topology on the $R$-module $\Omega_{R}^{*}$ and let $\widehat{\Omega}_{R}^{*}=\lim _{m} \Omega^{*} R / I^{m} \Omega_{R}^{*}$ be the corresponding completion. The de Rham differential $d^{m}: \Omega_{R}^{*} \longrightarrow \Omega_{R}^{*+1}$ is continuous and hence extends to a differential 
$\widehat{d}: \widehat{\Omega}_{R}^{*} \longrightarrow \widehat{\Omega}_{R}^{*+1}$. If $A$ is finitely generated the cohomology of $\left(\widehat{\Omega}_{R}, \widehat{d}\right)$ is independent of the presentation $A=R / I$, for $R$ smooth over $k$, and depends functorially on $A$ (cf. [13]); it coincides with the infinitesimal cohomology $H_{\text {inf }}(A)$ of $\operatorname{Spec} A$ over Speck, defined by Grothendieck in [12] as the correct generalization of de Rham cohomology for singular varieties.

The family of morphisms $\mu^{(i)}: \mathscr{B}_{\text {per }}^{(i)}\left(R / I^{m}\right) \longrightarrow \mathscr{D}_{\text {per }}^{(i)}\left(R / I^{m}\right), m \in \mathbf{N}$, induces a morphism

$$
\widehat{\mu}^{(i)}: \widehat{\mathscr{B}}_{\text {per }}^{(i)}(A ; R)=\varliminf_{m} \mathscr{B}_{\text {per }}^{(i)}\left(R / I^{m}\right) \longrightarrow \varliminf_{m}^{\lim } \mathscr{D}_{\text {per }}^{(i)}\left(R / I^{m}\right)=\widehat{\mathscr{D}}_{\text {per }}^{(i)}(A ; R) .
$$

Note that $\lim _{\leftarrow} \Omega_{R / I^{m}}^{*}=\lim _{m} \Omega_{R}^{*} / I^{m} \Omega_{R}^{*}$; hence, if $A=R / I$ is a smooth presentation of $A$, we have $H_{*}\left(\operatorname{Tot} \widehat{\mathscr{D}}_{\text {per }}^{(i)}(A ; R)\right)=H_{\text {inf }}^{2 i-*}(A)$.

Lemma 2.1 The projection $\widehat{\mathscr{B}}_{\mathrm{per}}^{(i)}(A ; R) \longrightarrow \mathscr{B}_{\mathrm{per}}^{(i)}(A)$ onto the component of degree $m=1$ is a quasi-isomorphism, i.e. $H_{*}\left(\operatorname{Tot} \widehat{\mathscr{B}}_{\mathrm{per}}^{(i)}(A ; R)\right) \simeq H P_{*}^{(i)}(A)$.

Proof. (cf. [24, Appl.1.15(2)]) This follows from the functoriality of the decomposition of periodic cyclic homology and Goodwillie's theorem on nilpotent ideals (cf. [10]).

Theorem 2.2 If $A$ is a commutative algebra of finite type there are canonical isomorphisms $\widehat{\mu}_{*}^{(i)}: H P_{*}^{(i)}(A) \longrightarrow H_{\mathrm{nff}}^{2 i-*}(A)$.

Proof. We can present $A$ as a quotient of a smooth algebra $R$ by some ideal $I \subseteq R$. Then $\widehat{\mu}^{(i)}: \widehat{\mathscr{B}}_{\text {per }}^{(i)}(A ; R) \longrightarrow \widehat{\mathscr{D}}_{\text {per }}^{(i)}(A ; R)$ induces in homology morphisms $\widehat{\mu}_{*}^{(i)}: H P_{*}^{(i)}(A) \longrightarrow H_{\mathrm{inf}}^{2 i-*}(A)$ via the identification of Lemma 2.1. Using standard arguments, one can show that these do not depend on the choice of the smooth presentation of $A$ and are functorial. Since the product morphism $\prod_{i \in \mathbf{Z}} \widehat{\mu}_{*}^{(i)}$ : $\Pi_{i \in \mathbf{Z}} H P_{*}^{(i)}(A) \longrightarrow \Pi_{i \in \mathbf{Z}} H_{\mathrm{inf}}^{2 i-*}(A)$ is an isomorphism (cf. $[8,27]$ ), we conclude that the same is true for $\widehat{\mu}_{*}^{(i)}$.

Remark 2.3 Using [14, Theorem 2], one can show that Theorem 2.2 holds more generally when $k$ is any commutative Noetherian ring containing $\mathbf{Q}$.

\subsection{The inverse system $\left(H C_{*+2 m}, S\right)_{m}, I$}

Let $A$ be a commutative algebra and consider for $n \in \mathbf{Z}$ the inverse system $\left(H C_{n+2 m}^{(n+m)}(A), S\right)_{m}$. We will denote by $\mathscr{H}_{n}(A)$ and $\mathscr{H}_{n}^{1}(A)$ the inverse limits $\lim _{m}$ $H C_{n+2 m}^{(n+m)}(A)$ and $\lim _{m}^{1} H C_{n+2 m}^{(n+m)}(A)$ respectively. The surjective inverse system of complexes $\left(\left(\overleftarrow{\operatorname{Tot}}_{\mathscr{B}}^{m} \mathscr{B}^{(i+m)}(A)\right)[-2 m], S\right)_{m}$ induces for all $* \in \mathbf{Z}$ functorial 
short exact sequences

$$
0 \longrightarrow \mathscr{H}_{2 i-*-1}^{1}(A) \longrightarrow H P_{*}^{(i)}(A) \longrightarrow \mathscr{H}_{2 i-*}(A) \longrightarrow 0 \text {. }
$$

If $A$ is finitely generated, we can use Theorem 2.2 and rewrite the above exact sequence as

$$
0 \longrightarrow \mathscr{H}_{n-1}^{1}(A) \longrightarrow H_{\text {inf }}^{n}(A) \longrightarrow \mathscr{H}_{n}(A) \longrightarrow 0, \quad n \in \mathbf{Z} .
$$

Theorem 2.4 Let $A$ be a commutative algebra of finite type.

(i) The inverse system $\left(H C_{n+2 m}^{(n+m)}(A), S\right)_{m}$ satisfies the Mittag-Leffler condition and hence $H_{\text {inf }}^{n}(A) \simeq \mathscr{H}_{n}(A)$.

(ii) The inverse system $\left(H C_{*+2 m}(A), S\right)_{m}$ satifies the Mittag-Leffler condition and hence $H P_{*}(A) \simeq \lim _{m} H C_{*+2 m}(A)$.

Proof. (i) The finite dimensionality of $H_{\text {inf }}^{n}(A)$ (cf. [13, Chap. II, Theorem 6.1]) and (5) imply that $\mathscr{H}_{n-1}^{1}(A)$ is finite dimensional. The result then follows from Proposition A.3 of the Appendix (Gray's criterion).

(ii) The first claim follows from (i); the second then follows from (3).

Remark 2.5 The conclusion of Theorem 2.4 may be false for an arbitrary ring $k$. Indeed, the periodic cyclic homology of the $\mathbf{Z}$-algebra $\mathbf{Z}[x] /\left(x^{n}-1\right), n \geqq 2$, is not the inverse limit of its cyclic homology (cf. [16]).

Let $A$ be a commutative algebra of finite type and consider the structural morphisms

$$
\sigma_{n}^{(l)}: H_{\mathrm{inf}}^{2 l-n}(A) \longrightarrow H C_{n}^{(l)}(A)
$$

of the inverse limit $H_{\mathrm{inf}}^{2 i-n}(A) \simeq \mathscr{H}_{2 i-n}(A)$. By their definition, these are compatible with $S$, i.e. $S \sigma_{n}^{(i)}=\sigma_{n-2}^{(i-1)}$. If $A=R / I$ is a presentation of $A$ as a quotient of a smooth algebra $R$, the projection $\widehat{\Omega}_{R}^{*}=\lim _{\leftarrow m} \Omega_{R / I^{m}}^{*} \longrightarrow \Omega_{A}^{*}$ onto the component of degree $m=1$ induces maps

$$
\Psi_{A}: H_{\mathrm{inf}}(A) \longrightarrow H_{\mathrm{dR}}(A) \text {. }
$$

One can verify that $\Psi_{A}$ does not depend on the choice of the smooth presentation of $A$ and is functorial.

Proposition 2.6 Let $A$ be a commutative algebra of finite type.

(i) If $i<n$ the composition $H_{\mathrm{inf}}^{2 i-n}(A) \stackrel{\sigma_{n}^{(i)}}{\longrightarrow} H C_{n}^{(i)}(A) \stackrel{\mu_{n}^{(i)}}{\longrightarrow} H_{\mathrm{dR}}^{2 l-n}(A)$ is the map $\Psi_{A}^{2 t-n}$, while the composition $H_{\mathrm{inf}}^{n}(A) \stackrel{\sigma_{n}^{(n)}}{\longrightarrow} H C_{n}^{(n)}(A) \stackrel{\mu_{n}^{(n)}}{\longrightarrow} \Omega_{A}^{n} / d \Omega_{A}^{n-1}$ coincides with $\Psi_{A}^{n}$ followed by the inclusion $j$ of $H_{\mathrm{dR}}^{n}(A)$ in $\Omega_{A}^{n} / d \Omega_{A}^{n-1}$.

(ii) $\sigma_{n}^{(i)}$ is injective for all $i \leqq n$.

Proof. (i) This follows since, given a presentation $A=R / I$ with $R$ smooth over $k$, the diagram below commutes for all $i<n$ 


$$
\begin{aligned}
& H_{n}\left(\operatorname{Tot} \widehat{\mathscr{B}}_{\text {per }}^{(i)}(A ; R)\right) \stackrel{\sim}{\rightarrow} H_{n}\left(\operatorname{Tot} \mathscr{B}_{\text {per }}^{(\prime)}(A)\right)=H P_{n}^{(i)}(A) \stackrel{\sim}{\rightarrow} \mathscr{H}_{2 i-n}(A) \\
& =\frac{\lim }{m} H C_{n+2 m}^{(i+m)}(A) \rightarrow H C_{n}^{(\imath)}(A) \\
& \widehat{\mu}_{n}^{(t)} \downarrow \simeq \\
& H_{\mathrm{inf}}^{2 i-n}(A) \longrightarrow \psi_{A}^{2 i-n} \longrightarrow H_{\mathrm{dR}}^{2 i-n}(A)
\end{aligned}
$$

(ii) Using the next theorem, this is a consequence of (i).

Theorem 2.7 (Deligne, Bloom, Herrera) If $A$ is a commutative algebra of finite type the map $\Psi_{A}: H_{\mathrm{mf}}(A) \longrightarrow H_{\mathrm{dR}}(A)$ is injective.

Proof. Assume that $k=\mathbf{C}$ and fix a smooth presentation $A=R / I$ of $A$. Let $Y=\operatorname{Spec} A, X=\operatorname{Spec} R$ and consider the associated analytic spaces $Y_{h}$ and $X_{h}$. Let $\Omega_{Y}$ (resp. $\Omega_{X}$ ) be the cochain complex of sheaves of algebraic differential forms on $Y$ (resp. X) and $\widehat{\Omega}_{\widehat{X}}$ the formal completion of $\Omega_{X}$ along the closed subscheme $Y$. Similarly, considering analytic differential forms on $Y_{h}$ and $X_{h}$, we obtain $\Omega_{Y_{h}}, \Omega_{X_{h}}$ and $\widehat{\Omega}_{\widehat{X}_{h}}$. Finally, let $\mathbf{C}_{Y_{h}}$ be the constant sheaf $\mathbf{C}$ on $Y_{h}$. We now consider the commutative diagram

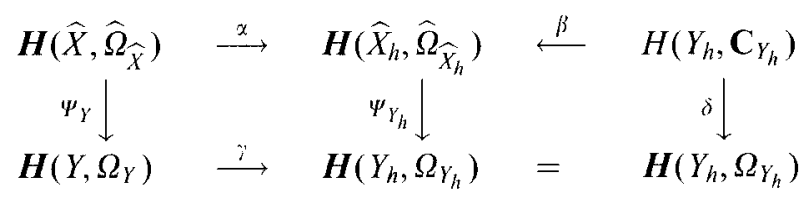

where $\mathbf{H}$ denotes hypercohomology, $\alpha$ and $\gamma$ are the comparison maps and $\beta$ (resp. $\delta$ ) is induced by the natural map $\mathbf{C}_{Y_{h}} \longrightarrow \widehat{\Omega}_{\widehat{X}_{h}}$ (resp. $\mathbf{C}_{Y_{h}} \longrightarrow \Omega_{Y_{h}}$ ). Then, $\alpha$ and $\beta$ are isomorphisms by [13, Chap. IV, Theorem 1.1] and $\delta$ is injective as shown in [2]. Hence, $\Psi_{Y}$ is injective. But $\Psi_{Y}$ is identified with $\Psi_{A}$ via the degeneracy of the corresponding hypercohomology spectral sequences.

The case of an arbitrary field $k$ (of characteristic 0 ) can be reduced to the one considered above using the compatibility of $H_{\mathrm{inf}}$ and $H_{\mathrm{dR}}$ with extensions of the base field (concerning $H_{1 \mathrm{ff}}$, see [13, Chap. III, Proposition 5.2]).

\section{The spectral sequence associated with Connes' exact couple}

\subsection{The spectral sequence associated with an exact couple}

Let $\mathscr{E}=(D, E, \alpha, \beta, \gamma)$ be an exact couple of vector spaces pictured as

$$
\begin{gathered}
D \stackrel{\alpha}{\longrightarrow} D \\
\mathrm{E}
\end{gathered}
$$


We will briefly review the construction of the spectral sequence associated with $\mathscr{E}$; for more details, the reader is refered to $[6,20]$. We first define a sequence $(\mathscr{E} r)_{r \geqslant 0}$ of exact couples (the higher derived couples of $\mathscr{E}=\mathscr{E}^{0}$ ), with $\mathscr{E}^{\mathscr{E}}=\left(D^{r}, E^{r}, \alpha^{(r)}, \beta^{(r)} \gamma^{(r)}\right)$ given by:

- $D^{r}=\alpha^{r} D=\operatorname{im}\left(D \stackrel{x^{r}}{\longrightarrow} D\right)$,

- $E^{r}=\gamma^{-1} \alpha^{r} D / \beta \alpha^{-r}(0)$, where $\alpha^{-r}(0)=\operatorname{ker}\left(D \stackrel{\alpha^{r}}{\longrightarrow} D\right)$,

- $\alpha^{(r)}$ is induced by $\alpha$,

- $\beta^{(r)}$ maps $\alpha^{r} d \in D^{r}$ onto the class $[\beta d] \in E^{r}$ and

- $\gamma^{(r)}$ maps a class $[e] \in E^{r}$ onto $\gamma e \in D^{r}$.

We now consider the endomorphism $\partial^{r}=\beta^{(r)} \gamma^{(r)}$ of $E^{r}$. Since $\left(\partial^{r}\right)^{2}=0$, $\left(E^{r}, \partial^{r}\right)$ is a differential vector space and one computes $H\left(E^{r}, \partial^{r}\right)=E^{r+1}$. It follows that $\left(\left(E^{r}, \partial^{r}\right)\right)_{r}$ is a spectral sequence of vector spaces. The limit term of the spectral sequence is given by $E^{\infty}=\gamma^{-1} \alpha^{\infty} D / \beta \alpha^{-\infty}(0)$, where $\alpha^{\infty} D=\cap_{r \geqq 0} \alpha^{r} D$ and $\alpha^{-\infty}(0)=\cup_{r \geqq 0} \alpha^{-r}(0)$. Hence, there is a short exact sequence

$$
0 \longrightarrow D /\left(\alpha^{-\infty}(0)+\alpha D\right) \stackrel{\beta}{\longrightarrow} E^{\infty} \stackrel{\gamma}{\longrightarrow} \alpha^{\infty} D \cap \alpha^{-1}(0) \longrightarrow 0 .
$$

Remark 3.1 (i) The differential $\partial^{r}$ of the spectral sequence associated with the exact couple $\mathscr{E}=(D, E, \alpha, \beta, \gamma)$ vanishes if and only if $\alpha^{r} D \cap \alpha^{-1}(0) \subseteq \alpha^{r+1} D$. It follows that the spectral sequence degenerates at $E^{r_{0}}$ if and only if the decreasing filtration of $\alpha^{-1}(0)=\operatorname{ker} \alpha$ by its subspaces $\alpha^{r} D \cap \alpha^{-1}(0)=\operatorname{ker} \alpha^{(r)}$, $r \geqq 0$, stabilizes at $r_{0}$, i.e. if and only if

$$
\alpha^{\infty} D \cap \alpha^{-1}(0)=\cdots=\alpha^{r_{0}+1} D \cap \alpha^{-1}(0)=\alpha^{r_{0}} D \cap \alpha^{-1}(0) .
$$

This happens if, for example, $\alpha^{r_{0}} D=\alpha^{r_{0}+1} D$.

(ii) Suppose that the exact couple $\mathscr{E}$ is graded by an abelian group $G$, i.e. $D$ and $E$ are $G$-graded vector spaces and $\alpha, \beta, \gamma$ are homogeneous morphisms of degrees $\operatorname{deg} \alpha, \operatorname{deg} \beta$ and $\operatorname{deg} \gamma$ respectively. Then $E^{r}, r \in \mathbf{N}$, and $E^{\infty}$ are also $G$-graded vector spaces and the differential $\partial^{r}$ of $E^{r}$ is homogeneous of degree $\operatorname{deg} \beta+\operatorname{deg} \gamma-r \operatorname{deg} \alpha$.

\subsection{The case of Connes' exact couple}

Let $A$ be a commutative algebra. Then, Connes' exact couple (2) is $\mathbf{Z} \oplus \mathbf{Z}$ graded (in the sense of Remark 3.1(ii)); in bidegree $(n, i), H C(A)$ and $H H(A)$ consist of $H C_{n}^{(i)}(A)$ and $H H_{n}^{(i)}(A)$ respectively and $\operatorname{deg} S=(-2,-1), \operatorname{deg} B=$ $(1,1)$ and $\operatorname{deg} I=(0,0)$. Therefore, the terms $E^{r}(A)$ of the spectral sequence and its limit term $E^{\infty}(A)$ are bigraded and the differential $\partial^{r}$ is homogeneous of degree $(1+2 r, 1+r)$.

For $r \geqq 1$, we have $E_{n}^{r(n)}(A)=\operatorname{im}\left(H C_{n+2 r}^{(n+r)}(A) \stackrel{S^{r}}{\longrightarrow} H C_{n}^{(n)}(A)\right)$. In particular, there is an isomorphism $E_{n}^{1(n)}(A) \simeq H_{\mathrm{dR}}^{n}(A)$ induced by the Loday-Quillen 
map $\mu$. Since $S$ is locally nilpotent, it follows from (6) that the limit term is given in bidegree $(n, i)$ by

$$
\begin{array}{r}
E_{n}^{\infty(i)}(A)=\left[\bigcap_{m \geqq 0} \operatorname{im}\left(H C_{n+2 m}^{(i+m)}(A) \stackrel{S^{m}}{\longrightarrow} H C_{n}^{(i)}(A)\right)\right] \\
\cap \operatorname{ker}\left(H C_{n}^{(i)}(A) \stackrel{S}{\longrightarrow} H C_{n-2}^{(i-1)}(A)\right) .
\end{array}
$$

Since $H C_{n-2}^{(n-1)}(A)=0$, we have:

$$
E_{n}^{\infty(n)}(A)=\bigcap_{m \geqq 0} \operatorname{im}\left(H C_{n+2 m}^{(n+m)}(A) \stackrel{S^{m}}{\longrightarrow} H C_{n}^{(n)}(A)\right) .
$$

The edge homomorphism $E_{n}^{\infty(n)}(A) \hookrightarrow E_{n}^{1(n)}(A)$ identifies $E_{n}^{\infty(n)}(A)$ with the subspace of $H_{\mathrm{dR}}^{n}(A)$ consisting of those elements which are annihilated by all higher order differentials of the spectral sequence

$$
E_{n}^{\infty(n)}(A) \subseteq \ldots \subseteq E_{n}^{2(n)}(A) \subseteq E_{n}^{\mid(n)}(A) \stackrel{\mu}{\simeq} H_{\mathrm{dR}}^{n}(A) .
$$

If $A$ is finitely generated the morphism $\sigma_{n}^{(n)}: H_{\text {inf }}^{n}(A) \longrightarrow H C_{n}^{(n)}(A)$ of Sect. 2.2 can be viewed as a map

$$
\tau_{n}: H_{\mathrm{inf}}^{n}(A) \longrightarrow E_{n}^{\infty(n)}(A) .
$$

Theorem 3.2 Let $A$ be a commutative algebra of finite type.

(i) $\tau_{n}: H_{\mathrm{inf}}^{n}(A) \longrightarrow E_{n}^{\infty(n)}(A)$ is an isomorphism for all $n$.

(ii) $E_{n}^{\infty(i)}(A)=0$ if $i<n$.

Proof. Using Theorem 2.4 and Proposition A.5 of the Appendix, we can rewrite (7) as

$$
E_{n}^{\infty(t)}(A)=\operatorname{im}\left(H_{\mathrm{inf}}^{2 i-n}(A) \stackrel{\sigma_{n}^{(i)}}{\longrightarrow} H C_{n}^{(i)}(A)\right) \cap \operatorname{ker}\left(H C_{n}^{(i)}(A) \stackrel{S}{\longrightarrow} H C_{n-2}^{(i-1)}(A)\right) .
$$

Therefore, assertions (i) and (ii) follow from Proposition 2.6(ii).

Remarks 3.3 (i) If $A$ is a commutative algebra of finite type the edge homomorphism $E_{n}^{\infty(n)}(A) \hookrightarrow E_{n}^{1(n)}(A)$ is identified with $\Psi_{A}^{n}: H_{\mathrm{nf}}^{n}(A) \longrightarrow$ $H_{\mathrm{dR}}^{n}(A)$.

(ii) If $A$ is an arbitrary (not necessarily commutative) algebra Connes' exact couple and the associated spectral sequence are $\mathbf{Z}$-graded. Up to grading, this spectral sequence is induced by the filtration of the double complex $\mathscr{B}_{\text {per }}(A)$ by columns.

If $A$ is a commutative algebra of finite type Theorem 3.2 implies that the limit term $E^{\infty}(A)=\bigoplus_{n} E_{n}^{\infty}(A)$ is isomorphic with $H_{\mathrm{inf}}(A)=\bigoplus_{n} H_{\mathrm{inf}}^{n}(A)$ as a $\mathrm{Z}$-graded vector space. If $*$ is the parity of $n$ and $\sigma_{n}: H P_{*}(A) \longrightarrow H C_{n}$ (A) the canonical map, there is an isomorphism $E_{n}^{\infty}(A) \simeq \operatorname{ker} \sigma_{n-2} / \operatorname{ker} \sigma_{n}$. 
(iii) Let $A=\bigoplus_{t \geq 0} A_{t}$ be a graded algebra. The induced grading on the tensor powers of $A$ is compatible with the differentials $b$ and $B$; hence, the double complex $\mathscr{B}(A)$ is itself graded as $\mathscr{B}(A)=\bigoplus_{1 \geq 0} \mathscr{B}(A)_{t}$, with $\mathscr{B}(A)_{0}=$ $\mathscr{B}\left(A_{0}\right)$. There results a decomposition of the exact couple $\mathscr{E}(A)$ as $\mathscr{E}(A)=$ $\bigoplus_{1 \geq 0} \mathscr{E}(A)_{t}$, with $\mathscr{E}(A)_{0}=\mathscr{E}\left(A_{0}\right)$, and hence a similar decomposition of the associated spectral sequence and its limit term. Using Goodwillie's theorem on the action of derivations in cyclic homology (cf. [10]), for the special case of the Euler field acting on $A$, one can show that the endomorphism $S$ of the exact couple $\mathscr{E}_{t}$ vanishes if $t \geqq 1$ (cf. [25, Proposition 2.4]). Hence, the inclusion $A_{0} \hookrightarrow A$ induces isomorphisms $E^{r}\left(A_{0}\right) \simeq E^{r}(A), r \geqq 1$, and $E^{\infty}\left(A_{0}\right) \simeq E^{\infty}(A)$.

Therefore, the spectral sequence associated with Connes' exact couple of a commutative algebra of finite type is (in degrees $r \geqq 1$ ) a homotopy invariant of the corresponding affine algebraic variety.

\subsection{The inverse system $\left(H C_{*+2 m}, S\right)_{m}, I I$}

Let $A$ be a commutative algebra of finite type and consider the kernel $V_{n}(A) \subseteq$ $H C_{n}(A)$ of the Loday Quillen map $\mu_{n}$. Then, $V(A)=\bigoplus_{n} V_{n}(A)$ is an $S$ invariant subspace of $H C(A)$. Let $V_{n}^{(i)}(A)=V(A) \cap H C_{n}^{(l)}(A)$.

Lemma 3.4 Let $A$ be a commutative algebra of finite type.

(i) There exists $n_{0}=n_{0}(A)$ such that for all $n, i$ with $n-i \geqq n_{0}$ there are canonical decompositions compatible with $S$

$$
H C_{n}^{(i)}(A)=H_{\mathrm{inf}}^{2 i-n}(A) \oplus V_{n}^{(i)}(A) .
$$

(ii) If $i<n$ there exists $m_{0}=m_{0}(i, n)$ such that for $m \geqq m_{0}$ we have

$$
\operatorname{ker}\left(H C_{n+2 m}^{(i+m)}(A) \stackrel{S^{m}}{\longrightarrow} H C_{n}^{(i)}(A)\right)=\operatorname{ker}\left(H C_{n+2 m}^{(i+m)}(A) \stackrel{S^{m+1}}{\longrightarrow} H C_{n-2}^{(l-1)}(A)\right) .
$$

(iii) If the spectral sequence associated with Connes' exact couple of $A$ degenerates at $E^{r_{0}}$ we can choose in (i) and (ii) above $n_{0}=r_{0}=m_{0}(i, n)$ for all $i<n$.

Proof. (i) Theorems 2.4 and 3.2 imply that there exists $n_{0} \geqq 0$ such that for all $n$

$$
\operatorname{im}\left(H C_{n+2 m}^{(n+m)}(A) \stackrel{S^{m}}{\longrightarrow} H C_{n}^{(n)}(A)\right) \stackrel{\mu}{\simeq} H_{\mathrm{inf}}^{n}(A)
$$

when $m \geqq n_{0}$ (note that $H C_{n}^{(n)}(A)$ and $H_{\text {inf }}^{n}(A)$ vanish for all but finitely many $n$ 's). Proposition 2.6(i) shows that, if $m \geqq n_{0}$ and $m>0$, the surjection of $H C_{n+2 m}^{(n+m)}(A)$ onto the image $H_{\text {inf }}^{n}(A)$ of $\mu_{n}^{(n)} S^{m}=j \mu_{n+2 m}^{(n+m)}$ (cf. (4)) admits a canonical splitting. If $m=n_{0}=0$ we have $H C_{n}^{(n)}(A) \stackrel{\mu}{\simeq} H_{\text {inf }}^{n}(A)$ and hence (8) holds for $i=n$. 
(ii) Given $i<n$, Theorems 2.4 and 3.2 imply that there exists $m_{0}=$ $m_{0}(i, n) \geqq 0$ such that

$$
\operatorname{im}\left(H C_{n+2 m}^{(i+m)}(A) \stackrel{S^{m}}{\longrightarrow} H C_{n}^{(i)}(A)\right) \cap \operatorname{ker}\left(H C_{n}^{(i)}(A) \stackrel{s}{\longrightarrow} H C_{n-2}^{(1-1)}(A)\right)=0
$$

for $m \geqq m_{0}$. The above equality is equivalent to (9).

(iii) This follows from the proofs given above and Remark 3.1(i).

Theorem 3.5 Let A be a commutative algebra of finite type.

(i) There exists $n_{0}=n_{0}(A)$ such that the inverse system $\left(H C_{n+2 m}^{(n+m)}\right.$ $(A), S)_{m \geqq n_{0}}$ decomposes for all $n$ into the direct sum of the constant system with value $H_{\mathrm{nff}}^{n}(A)$ and the system $\left(V_{n+2 m}^{(n+m)}(A), S\right)_{m \geqq n_{0}}$, which is essentially zero.

(ii) There exists $N_{0}=N_{0}(A)$ such that the inverse system $\left(H C_{*+2 m}\right.$ (A), $S)_{m \geqq N_{0}}$ decomposes for $*=0,1$ into the direct sum of the constant system with value $\bigoplus_{l \in \mathbf{Z}} H_{\mathrm{inf}}^{*+2 l}(A)$ and the system $\left(V_{*+2 m}(A), S\right)_{m \geqq N_{0}}$, which is essentially zero.

Proof. (i) The first claim is a reformulation of Lemma 3.4(i). Theorem 2.4 implies that the system $\left(V_{n+2 m}^{(n+m)}(A), S\right)_{m}$ satisfies the Mittag-Leffler condition and $\lim _{m} V_{n+2 m}^{(n+m)}(A)=0$, whence the last claim.

(ii) If $A$ can be generated as a $k$-algebra by $g(>0)$ elements and $n_{0} \geqq 0$ is as above, we consider $N_{0}^{\prime}=2 n_{0}+g-2$. For all $n \geqq N_{0}^{\prime}$ we then have $H C_{n}^{(i)}(A)=H_{\text {inf }}^{2 i-n}(A) \oplus V_{n}^{(i)}(A)$. If $n-i \geqq n_{0}$, this follows from (i). On the other hand, if $n \geqq N_{0}^{\prime}$ and $n-i<n_{0}$, both sides of the equality vanish since $2 i-n \geqq g$ (the vanishing of $H C_{n}^{(i)}(A)$ is shown in [26] and that of $H_{\text {inf }}^{2 i-n}(A)$ follows since $\left.H_{\mathrm{dR}}^{2 i-n}(A)=0\right)$. Hence, we can choose $N_{0}=\left[\left(N_{0}^{\prime}+1\right) / 2\right]$. Using Theorem 2.4 as above, we conclude that the system $\left(V_{*+2 m}(A), S\right)_{m}$ is essentially zero.

Remark 3.6 The decomposition of Theorem 3.5 is canonical in the following sense. If $B$ is another algebra of finite type and $f: A \longrightarrow B$ an algebra morphism, the induced map $f: H C_{n}(A) \longrightarrow H C_{n}(B)$ splits when $n \geqq$ $\max \left(N_{0}^{\prime}(A), N_{0}^{\prime}(B)\right)$ into the direct sum of the morphisms $f: V_{n}(A) \longrightarrow V_{n}(B)$ and $f: H_{\mathrm{inf}}^{n+2 l}(A) \longrightarrow H_{\mathrm{inf}}^{n+2 l}(B), l \in \mathbf{Z}$.

\subsection{Degeneracy criteria}

Let $A$ be a commutative algebra of finite type. In this section, we study the relation between the restriction of the operator $S$ on the kernel $V(A)$ of the Loday-Quillen map and the degeneracy of the spectral sequence associated with Connes' exact couple of $A$. We note that Theorem 3.5 implies that for any $n$ there exists $m=m(n)$ such that the map $S^{m}: V_{n+2 m}(A) \longrightarrow V_{n}(A)$ vanishes. 
Theorem 3.7 Let $A$ be a commutative algebra of finite type and consider the spectral sequence associated with Connes' exact couple of $A$. ishes.

(i) If the spectral sequence degenerates at $E^{r_{0}}$ the restriction $\left.S^{r_{0}}\right|_{V(A)}$ van-

(ii) Conversely, if $\left.S\right|_{V(A)}$ is nilpotent the spectral sequence degenerates.

Proof. (i) If the spectral sequence degenerates at $E^{r_{0}}$ Lemma 3.4(iii) shows that (9) is valid for all $i<n$ and $m \geqq r_{0}$. Applying that equation repeatedly, we conclude that

$$
\operatorname{ker}\left(H C_{n}^{(i)}(A) \stackrel{S^{\prime 0}}{\longrightarrow} H C_{n-2 r_{0}}^{\left(i-r_{0}\right)}(A)\right)=\operatorname{ker}\left(H C_{n}^{(i)}(A) \stackrel{S^{n-1}}{\longrightarrow} H C_{2 i-n}^{(2 \imath-n)}(A)\right)=V_{n}^{(i)}
$$

for $n-i \geqq r_{0}$, where the second equality follows from the commutativity of diagram (4). Hence, $S^{r_{0}} V_{n}^{(i)}(A)=0$ for $n-i \geqq r_{0}$. Since $H C_{n-2 r_{0}}^{\left(i-r_{0}\right)}(A)=0$ if $n-i<r_{0}$, we always have $S^{r_{0}} V_{n}^{(i)}(A)=0$.

(ii) Let $S^{N}$ vanish on $V(A)$, where $N$ is large enough so that (8) is valid for $n-i \geqq N$. If $m \geqq N$ we compute

$$
\begin{gathered}
\operatorname{im}\left(H C_{n+2 m}^{(i+m)}(A) \stackrel{S^{m}}{\longrightarrow} H C_{n}^{(i)}(A)\right) \cap \operatorname{ker}\left(H C_{n}^{(i)}(A) \stackrel{S}{\longrightarrow} H C_{n-2}^{(i-1)}(A)\right) \\
=\left\{\begin{array}{cl}
H_{\text {inf }}^{n}(A) & \text { if } i=n \\
0 & \text { if } i<n .
\end{array}\right.
\end{gathered}
$$

Theorem 3.2 and Remark 3.1(i) imply that the spectral sequence degenerates at $E^{N}$.

Now, we want to characterize those commutative algebras of finite type for which the spectral sequence in question degenerates at $E^{1}$. If $A$ is such an algebra the decomposition of Theorem 3.5 (ii) holds in degrees $\geqq g$, the minimal number of generators of $A$; this follows from Lemma 3.4(iii) and the proof of Theorem 3.5.

Definition $3.8([3,15])$ A commutative algebra $A$ of finite type has property (P) if there exist graded vector spaces $U=\bigoplus_{n \geqq 0} U_{n}$ and $V^{\prime}=\bigoplus_{n \geqq 0} V_{n}^{\prime}$, such that:

(i) For all $n, H C_{n}(A)=V_{n}^{\prime} \oplus U_{n} \oplus U_{n-2} \oplus U_{n-4} \oplus \cdots$ and

(ii) The periodicity operator $S: H C_{n}(A) \longrightarrow H C_{n-2}(A)$ vanishes on $V_{n}^{\prime} \oplus U_{n}$ and maps identically $U_{n-2} \oplus U_{n-4} \oplus \cdots \subseteq H C_{n}(A)$ onto the corresponding summand of $\mathrm{HC}_{n-2}(A)$.

Remarks 3.9 (i) Consider the polynomial algebra $k[u]$ graded by deg $u=2$. Then, $u^{n} \mapsto \sum_{i=0}^{n} u^{i} \otimes u^{n-i}$ induces a coalgebra structure on $k[u]$ and the periodicity operator $S$ induces the structure of a $k[u]$-comodule on the cyclic homology $H C(A)$ of $A$. The algebra $A$ has property $(\mathrm{P})$ if this comodule decomposes into the direct sum of a trivial comodule $V^{\prime}$ and an extended comodule $U \otimes k[u]$. 
(ii) If $A$ has property (P) the graded space $U$ in the above definition is given in degree $n$ by $U_{n}=E_{n}^{\infty}(A) \simeq H_{\text {inf }}^{n}(A)$ (cf. Remark 3.3(ii)). Hence, $U$ is finite dimensional.

Theorem 3.10 For a commutative algebra $A$ of finite type, the following are equivalent:

(i) The spectral sequence associated with Connes' exact couple of A degenerates at $E^{1}$.

(ii) $E_{n}^{l(l)}(A)=0$ for $i<n$.

(iii) $\operatorname{im}(H C(A) \stackrel{s}{\longrightarrow} H C(A))=\operatorname{im}\left(H C(A) \stackrel{s^{2}}{\longrightarrow} H C(A)\right)$.

(iv) $A$ has property $(P)$.

If these conditions are satisfied then:

$(\alpha)$ The canonical map $\Psi_{A}: H_{\mathrm{inf}}(A) \longrightarrow H_{\mathrm{dR}}(A)$ is an isomorphism.

( $\beta$ ) There is a short exact sequence

$$
0 \longrightarrow V(A) \longrightarrow H C(A) \stackrel{\mu}{\longrightarrow} H(\operatorname{Tot} \mathscr{D}(A)) \longrightarrow 0,
$$

which admits a canonical splitting compatible with the operator $S$.

$(\gamma)$ For all $i<n$ there are short exact sequences

$$
0 \longrightarrow V_{n-1}^{(i-1)}(A) \stackrel{B}{\longrightarrow} H H_{n}^{(i)}(A) \stackrel{I}{\longrightarrow} V_{n}^{(i)}(A) \longrightarrow 0,
$$

which make explicit the acyclicity of $\left(H H_{*}^{(*-l)}(A), B I\right), l>0$.

Proof. (i) $\longrightarrow$ (ii) $E_{n}^{(1)}(A)=E_{n}^{\infty(t)}(A)=0$ for $i<n$, by Theorem 3.2.

(ii) $\longrightarrow$ (iii) Let $y \in \operatorname{im} S \cap H C_{n}^{(i)}(A)$ for some $i \leqq n$ and choose $x \in$ $H C_{n+2}^{(i+1)}(A)$ such that $y=S x$. Since $B I(B x)=0$ and $E_{n+3}^{1(i+2)}(A)=0$, we can choose $z \in H H_{n+2}^{(l+1)}(A)$ such that $B x=B I z$. But then $x-I z \in \operatorname{ker} B=\operatorname{im} S$ and hence $y=S x=S(x-I z) \in \operatorname{im} S^{2}$.

(iii) $\rightarrow$ (i) This follows from Remark 3.1(i).

(iv) $\longrightarrow$ (iii) This follows from the definition of property (P).

(i) $\longrightarrow$ (iv) We know from Remark 3.3(i) that $\Psi_{A}: H_{\mathrm{inf}}(A) \longrightarrow H_{\mathrm{dR}}(A)$ is an isomorphism. Hence (8), which now holds for all $0 \leqq i<n$, shows that $\mu_{n}^{(i)}$ is a canonically split surjection for $i<n$ (recall that $\mu_{n}^{(n)}$ is an isomorphism). Moreover, the splittings are compatible with $S$. Theorem 3.7 implies that $S$ vanishes on the kernel of $\mu$; it follows that $A$ has property (P).

Since $S$ vanishes on $V(A),(8)$ implies that

$$
V_{n}^{(l)}(A)=\operatorname{ker}\left(H C_{n}^{(i)}(A) \stackrel{S}{\longrightarrow} H C_{n-2}^{(i-1)}(A)\right)=\operatorname{im}\left(H H_{n}^{(i)}(A) \stackrel{I}{\longrightarrow} H C_{n}^{(l)}(A)\right)
$$

and

$$
\begin{gathered}
V_{n-1}^{(i-1)}(A)=\operatorname{coker}\left(H C_{n+1}^{(1)}(A) \stackrel{S}{\longrightarrow} H C_{n-1}^{(i-1)}(A)\right) \\
\stackrel{B}{\simeq} \operatorname{im}\left(H C_{n-1}^{(1-1)}(A) \stackrel{B}{\longrightarrow} H H_{n}^{(i)}(A)\right)
\end{gathered}
$$

for $i<n$, whence the last assertion of the theorem. 
Remarks 3.11 (i) Smooth algebras satisfy the equivalent conditions of Theorem 3.10. More generally, if $A=\bigoplus_{t \geqq 0} A_{t}$ is a graded algebra with $A_{0}$ smooth, then $A$ satisfies these conditions as well. In particular, there are non-smooth algebras satisfying these conditions. On the other hand, since the canonical map $\Psi$ is not always an isomorphism, there are commutative algebras of finite type which do not satisfy these conditions.

(ii) Let $A$ be a commutative algebra of finite type such that the spectral sequence associated with Connes' exact couple of $A$ degenerates at $E^{0}$. Then, $A$ is smooth over $k$; this follows from [1] since $H H_{n}^{(i)}(A)=E_{n}^{0(t)}(A)=$ $E_{n}^{\infty(1)}(A)=0$ if $i \neq n$. In particular, $A$ is reduced. Since $A=H H_{0}(A)=E_{0}^{0}(A)=$ $E_{0}^{\infty}(A)=H_{\text {inf }}^{0}(A)$ is a finite dimensional vector space, we conclude that $A$ is a finite direct product of finite field extensions of $k$.

Conversely, if $A$ is such a direct product the system $\left(H C_{*+2 m}\right.$ $(A), S)_{m}$ is constant with value $A$ (resp. 0 ) if $*=0$ (resp. 1). In particular, the operator $S$ is surjective and hence Remark 3.1(i) implies that the spectral sequence associated with Connes' exact couple of $A$ degenerates at $E^{0}$.

\section{Appendix: Inverse limits}

In this Appendix, we prove Propositions A.3 and A.5, which were already used in the proofs of Theorems 2.4 and 3.2. Proposition A.3 (Gray's criterion) was proved in [11], when $k=\mathbf{Z}$. Considering dimension instead of cardinality, we will present Gray's arguments for the case of a field. The result of Proposition A.5 is folklore.

We consider inverse systems of vector spaces indexed by the ordered set $\mathbf{N}$. The higher inverse limit functors $\lim ^{\prime}$ vanish if $i \geqq 2$ (cf. [23]); hence, a short exact sequence of inverse systems

$$
0 \longrightarrow\left(X_{m}^{\prime}\right)_{m} \longrightarrow\left(X_{m}\right)_{m} \longrightarrow\left(X_{m}^{\prime \prime}\right)_{m} \longrightarrow 0
$$

induces a 6-term exact sequence

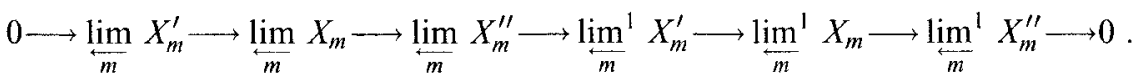

Let $\left(X_{m}\right)_{m}$ be an inverse system and consider, for $n \in \mathbf{N}$, the system $\left(X_{m}^{n}\right)_{m}$ defined by

$$
X_{m}^{n}= \begin{cases}\operatorname{im}\left(X_{m} \longrightarrow X_{n}\right) & \text { if } m \geqq n \\ 0 & \text { if } m<n\end{cases}
$$

Let $\widehat{X}_{n}=\lim _{m \geq n} X_{n} / \mathrm{im}\left(X_{m} \longrightarrow X_{n}\right)$ be the completion of $X_{n}$ with respect to the filtration of it by the images of $X_{m}, m \geqq n$, and $\theta_{n}: X_{n} \longrightarrow \widehat{X}_{n}$ the canonical map. Using the 6-term exact sequence (10), one can show that $\lim _{m}^{1} X_{m}^{n}=$ $\operatorname{coker} \theta_{n}$.

Lemma A.1 If $\left(X_{m}\right)_{m}$ is an inverse system and $n \in \mathbf{N}$, there exists a functorial surjective morphism $\lim _{m}^{1} X_{m} \longrightarrow \operatorname{coker} \theta_{n}$. 
Proof: There is a canonical epimorphism of inverse systems $\left(X_{m}\right)_{m} \longrightarrow$ $\left(X_{m}^{n}\right)_{m}$. Since $\lim _{\leftarrow}^{\prime}$ is right exact, it induces a surjective map of vector spaces.

Lemma A.2 Let $F$ be a vector space filtered by a sequence $F=F_{0} \supseteq F_{1} \supseteq$ $F_{2} \supseteq \cdots$ of subspaces. Then, if the completion $\hat{F}=\lim _{m} F / F_{m}$ is countably generated the filtration is eventually constant.

Proof. Fix decompositions $F_{m-1}=F_{m} \oplus G_{m}, m>0$, and suppose that $G_{m} \neq \mathbf{0}$ for infinitely many $m$ 's. Then, we can construct an uncountable linearly independent family of vectors in $\hat{F}=\Pi_{m>0} G_{m}$ using an uncountable chain of distinct non-empty subsets of $\mathbf{N}$.

Proposition A.3 (Gray) If $\left(X_{m}\right)_{m}$ is an inverse system of countably generated vector spaces the following conditions are equivalent:

(i) The inverse system $\left(X_{m}\right)_{m}$ satisfies the Mittag-Leffler condition.

(ii) $\lim _{m}^{1} X_{m}=0$.

(iii) $\overleftarrow{\lim }_{m}^{1} X_{m}$ is countably generated

Proof. (iii) $\rightarrow$ (i). Fix $n \in \mathbf{N}$ and note that coker $\left(X_{n} \stackrel{\theta_{n}}{\longrightarrow} \widehat{X}_{n}\right)$ is countably generated by Lemma A.1. Since so is $X_{n}$, it follows that $\widehat{X}_{n}$ is countably generated as well. Hence, we can apply Lemma A.2 and conclude that the filtration of $X_{n}$ by the images of $X_{m}, m \geqq n$, is eventually constant. This being true for all $n$, the inverse system $\left(X_{m}\right)_{m}$ satisfies the Mittag-Leffler condition, as needed.

Remark A.4 If $\left(X_{m}\right)_{m}$ is an arbitrary inverse system there is no control on the dimension of $\lim _{\leftarrow m}^{1} X_{m}$, as the following example shows.

Given a vector space $M$, consider the inclusion $M^{(\mathbf{N})} \hookrightarrow M^{\mathbf{N}}$ of the countable direct sum of copies of $M$ into the corresponding direct product. Let $X$ be a subspace of $M^{\mathrm{N}}$ containing $M^{(\mathrm{N})}$ such that $M^{\mathrm{N}} / X \simeq M$ (such a subspace exists, since the image of the diagonal embedding of $M$ into $M^{\mathrm{N}}$ intersects $M^{(\mathrm{N})}$ trivially). Let $F_{m} \subseteq M^{\mathrm{N}}$ consist of those sequences in $M$ whose first $m$ entries are 0 . Consider the inverse system $\left(X_{m}\right)_{m}$ with $X_{m}=X \cap F_{m}$ and structural morphisms the inclusions. The completion $\widehat{X}=\lim _{m} X_{0} / X_{m}$ of $X=X_{0}$ is identified with $M^{\mathbf{N}}$ in such a way that the canonical morphism $\theta: X \longrightarrow \hat{X}$ coincides with the inclusion $X \hookrightarrow M^{\mathrm{N}}$. Hence, $\lim _{m}^{\prime} X_{m}=M^{\mathrm{N}} / X \simeq M$.

Now let $\left(X_{m}\right)_{m}$ be an inverse system and consider the system $\left(Y_{m}\right)_{m}$ given by

$$
Y_{m}=\bigcap_{m^{\prime} \geqq m} \operatorname{im}\left(X_{m^{\prime}} \longrightarrow X_{m}\right) .
$$

Then, the inclusions $Y_{m} \subseteq X_{m}$ induce an isomorphism $\lim _{m} Y_{m} \simeq \lim _{m} X_{m}$. If the inverse system $\left(X_{m}\right)_{m}$ satisfies the Mittag-Leffler condition the system $\left(Y_{m}\right)_{m}$ is surjective. 
Proposition A.5 If the inverse system $\left(X_{m}\right)_{m}$ satisfies the Mittag-Leffler condition the canonical morphism $p_{0}: \lim _{m} X_{m} \longrightarrow X_{0}$ maps $\lim _{m} X_{m}$ onto $\cap_{m \geqq 0} \operatorname{im}\left(X_{m} \longrightarrow X_{0}\right)$.

Proof. Considering the inverse system of stable images as above, we reduce to the case of a surjective system. In that case, the result is straightforward.

Remark A.6 Without some assumption on the inverse system $\left(X_{m}\right)_{m}$, the image of the canonical map $p_{0}: \lim _{m} X_{m} \longrightarrow X_{0}$ may be strictly contained in $\cap_{m \geqq 0} \operatorname{im}\left(X_{m} \longrightarrow X_{0}\right)$. In fact, for any morphism $f: M \longrightarrow N$ there exists an inverse system $\left(X_{m}\right)_{m}$ with $\lim _{\leftarrow} X_{m}=M$ and $\cap_{m \geqq 0} \operatorname{im}\left(X_{m} \longrightarrow X_{0}\right)=N$ such that the map $p_{0}$ is identified with $f$. The following example is a variant of $[6$, Example 6.11].

Let $W$ be the free abelian group with basis $\left\{e_{m}: m=1,2, \ldots\right\}, \varepsilon: W \longrightarrow \mathbf{Z}$ the augmentation with $e_{m} \mapsto 1$ and $T$ the endomorphism of $W$ given by $e_{m} \mapsto$ $e_{m+1}$. Then, the inverse system $\left(X_{m}\right)_{m}$ with $X_{m}=\left(N \otimes_{\mathbf{z}} W\right) \oplus M$ for $m \geqq 1$ and $X_{0}=N$, whose structural morphisms $X_{m} \longrightarrow X_{m-1}$ are given by $\left(i d_{N} \otimes T\right) \oplus i d_{M}$ for $m>1$ and the matrix $\left(i d_{N} \otimes \varepsilon f\right.$ ) for $m=1$, has the required properties.

Acknowledgements. The results of this paper were obtained as part of the author's Ph.D. thesis at the University of California at Berkeley. I would like to express my deep gratitude to my thesis advisor Mariusz Wodzicki for his warm support and encouragement.

\section{References}

1. Avramov, L., Vigué-Poirrier, M.: Hochschild homology criteria for smoothness. I.R.M.N. Duke Math. J. 65, 17-25 (1992)

2. Bloom, T., Herrera, M.: De Rham cohomology of an analytic space. Invent. Math. 7, 275-296 (1969)

3. Burghelea, D., Ogle, C.: The Künneth Formula in Cyclic Homology. Math. Z. 193, 527-536 (1986)

4. Burghelea, D., Vigué-Poirrier, M.: Cyclic homology of commutative algebras. In: Proc. Meeting on Algebraic Homotopy, Louvain, 1986 (Lect. Notes in Mathematics, vol. 1318, pp. 51-72) Berlin Heidelberg New York: Springer 1988

5. Connes, A.: Non commutative differential geometry. Publ. Math. IHES 62, 41-144 (1985)

6. Eckmann, B., Hilton, P.J.: Exact couples in an abelian category. J. Algebra 3, 38-87 (1966)

7. Emmanouil, I.: Cyclic homology and de Rham homology of commutative algebras. C.R. Acad. Sci. Paris 318, 413-417 (1994)

8. Feigin, B.L., Tsygan, B.L.: Additive K-theory and crystalline cohomology. Funct. Anal. Appl. 19, 124-132 (1985)

9. Gerstenhaber, M., Schack, S.D.: A Hodge-type decomposition for commutative algebra cohomology. J. Pure Appl. Algebra 48, 229-247 (1987)

10. Goodwillie, T.: Cyclic homology, derivations and the free loopspace. Topology 24, $187-215(1985)$

11. Gray, B.: Spaces of the same $n$-type, for all $n$. Topology 5, 241-243 (1966)

12. Grothendieck, A.: Crystals and the de Rham cohomology of schemes, notes by J. Coates and D. Jussila. Dix exposés sur la cohomologie des schémas, Amsterdam: North-Holland, 1968 
13. Hartshorne, R.: On the de Rham cohomology of algebraic varieties. Publ. Math. IHES 45, 5-99 (1976)

14. Hübl, R.: A note on the Hochschild homology and cyclic homology of a topological algebra. Manus. Math. 77, 63-70 (1992)

15. Kassel, C.: Cyclic homology, comodules and mixed complexes. J. Algebra 107, 195-216 (1987)

16. Kassel, C.: Quand 1'homologie cyclique périodique n'est pas la limite projective de l'homologie cyclique. K-Theory 2, 617-621 (1989)

17. Loday, J.-L.: Opérations sur I'homologie cyclique des algébres commutatives. Invent. Math. 96, 205-230 (1989)

18. Loday, J.-L.: Cyclic Homology. (Grundl. Math. Wiss. 301) Berlin Heidelberg New York: Springer 1992

19. Loday, J.-L., Quillen, D.: Cyclic homology and the Lie algebra homology of matrices. Comment. Math. Helv. 59, 565-591 (1984)

20. MacLane, S.: Homology. (Grundl. Math. Wiss. 114) Berlin Heidelberg New York: Springer 1963

21. Natsume, T., Schack, S.D.: A decomposition for the cyclic cohomology of a commutative algebra. J. Pure Appl. Algebra 61, 273-282 (1989)

22. Ronco, M.: On the Hochschild homology decompositions. Comm. Alg. 21, 4699-4712 (1993)

23. Roos, J.E.: Sur les foncteurs dérivés de $\lim _{\longleftarrow}$. Applications. C.R. Acad. Sci. Paris 252, 3702-3704 (1961)

24. Seibt, P.: Local cyclic homology. K-Theory 4, 143-155 (1990)

25. Vigué-Poirrier, M.: Cyclic homology of algebraic hypersurfaces. J. Pure Appl. Alg. 72, 95-108 (1991)

26. Vigué-Poirrier, M.: Décompositions de 1 'homologie cyclique des algébres différentielles graduées commutatives. K-Theory 4, 399-410 (1991)

27. Wodzicki, M.: Periodic cyclic homology of affine algebras, unpublished lecture notes, June 1989 